\title{
HETERONYM VERIFICATION FOR MANDARIN SPEECH SYNTHESIS
}

\author{
Lu Heng, Ling Zhenhua, Wei Si, Hu Yu, Dai Lirong, Wang Renhua \\ iFlytek Speech Lab, University of Science and Technology of China, Hefei, Anhui
}

\begin{abstract}
Accurate phonetic transcription of speech corpus is critical to high quality speech synthesis. In Mandarin text-to-speech (MTTS) system, one major problem of automatically labeling the database is the heteronym annotation. Because in Mandarin, there are some single-character words or multi-character words have more than one pronunciation. In this paper, a heteronym annotation verification method for MTTS database labeling is proposed. By training contextual dependent HMMs and calculating the log likelihood ratio, each heteronym in the database is assigned a confidence score and those below the threshold are selected for manual inspecting. We divide heteronyms in Mandarin into two categories and different features are used for each category. The result of our experiment on an artificial test set has shown that we can achieve EER (equal error rate) of $7.9 \%$ and $11.9 \%$ for these two categories. Further test on an actual database which contains a total of 36098 heteronyms has shown that the proposed method can find 89 of all 123 annotation errors by only inspecting 639 polyphones.
\end{abstract}

Index Terms - heteronym annotation, log likelihood ratio, MTTS, , automatic labeling

\section{INTRODUCTION}

In speech synthesis, the quality of the synthesized speech counts much on the annotation of the database. A welllabeled database is the precondition for accurate acoustic model estimation and reliable unit selection. Traditional annotation method requires lots of manual works either by labeling the database by hand or by checking the automatic annotation manually. We want to make the process of labeling a Mandarin database as automatic as possible and reduce the manual work but still achieve high quality.

In Mandarin, there are some single-character words or multi-character words that have more than one pronunciation, named "heteronym". The heteronym problem is a particular issue of MTTS [1]. Besides polyphonic words, there is no out-of-vocabulary problem in characterto-syllable conversion in Mandarin. Any new word can be converted simply by looking-up a character-to-syllable dictionary. To tackle the heteronym problem, listing as many words with polyphonic characters as possible, such as “中 (zhong4)奖” and “行 (hang2) 长 (zhang3)", into the dictionary is an approach commonly-employed in most MTTS systems. But there are still $0.4 \%-0.9 \%$ automatic labeling errors which may affect the quality of the synthesized speech.

In this paper, we proposed to locate the heteronym annotation errors from the annotation verification view, and use log likelihood ratio [2] of various features to locate the heteronym annotation errors in a Mandarin speech synthesis database. For that Mandarin is a tonal language, and heteronym can be pronounced differently either in the phone itself or just in tone, we divide the heteronyms into 2 categories: class $\mathrm{A}$ is the heteronym pronounced differently just in tone, and class B is the ones pronounced different in phone and either the same or different in tone. Different features are used to calculate the confidence score for each category. Besides, instead of using phone, bi-phone or triphone model, contextual dependent HMMs are used for the annotation verification. What is more, all the context information can be obtained from the speech synthesis database and no more data preparation work for annotation verification needed.

Section 2 firstly gives an overview of the main frame of the annotation verification system. Then it introduces the proposed context information dependent tri-phone HMM model for annotation verification, the log likelihood ratio of different acoustic features, the two classes of the heteronym and the spectral and f0 acoustic features used to verify each of the classes. Section 3 shows an experiment. And finally, section 4 and section 5 presents conclusions and feature works.

\section{HETERONYM ANNOTATION VERIFICATION SYSTEM}

\subsection{System overview}

Figure 1 gives an overview of the heteronym annotation verification system. The whole system can be divided into two parts: the contextual dependent HMM training part, and the annotation verification part which includes the log likelihood ratio confidence measure calculation and the error decision for the two class heteronyms.

\subsection{Context dependent tri-phone HMM model}




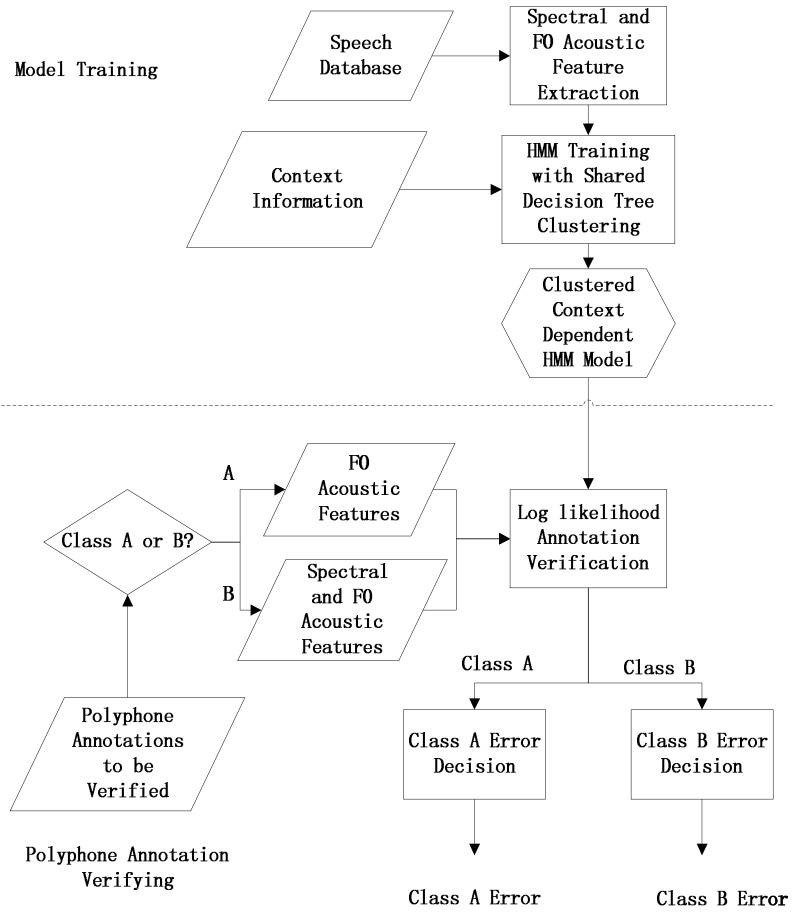

Figure 1: Flow chart of proposed method

Unlike speech recognition tasks, in annotation verification of the speech synthesis database, lots of context information can be obtained from the database, because speech synthesis counts much on accurate context information. Such information can also be employed to give rise to the accuracy of the annotation verification system.

In our method, we select the context information dependent tri-phone HMM model as our model for annotation verifying for two reasons:

- Mandarin is a tonal language, and there are lots of coarticulation phenomena in the continuous speech of Mandarin, especially in tone [3]. With more specific context information, more accurate models can be trained, which can give rise to the verification quality.

- In speech synthesis database, unlike tasks in speech recognition, labels are annotated with various context information that are necessary in speech synthesis. We can use the same context information for annotation verification without extra effort in labeling. Furthermore, if the speech synthesis system is HMM based, the same HMM model training steps can be used for training the model for annotation verification, or even the same person's model trained last time for synthesis can be used for label verification. This simplifies the construction of an annotation verification system.

In our label for both speech synthesis and annotation verification, we include such context information as below:

- Left phone : phone before the current phone

- Current phone : the phone that we are verifying

- Right phone : phone after the current phone
- Left tone : tone of the syllable before the current syllable

- Current tone : the tone that we are verifying

- Right tone : tone of the syllable after the current syllable

- Part-of-speech : nature of the current word

- Relative positions of current syllable, word, phrase, sentence, sentence group

- Absolute positions from head and tail of current syllable, word, phrase, sentence, sentence group

Since the combination of these features may result in a large number of context models, decision-tree-based model clustering using the minimum description length (MDL) [4] criterion is introduced. The purpose of model clustering is to avoid the over-fitting problem and, at the same time, to predict the acoustic model for the out-of-set annotation.

\subsection{Log likelihood ratio}

Generalized posterior probability (GPP) is the confidence measure commonly used in ASR for assessing the ASR reliability. And previous researches had shown GPP is an effective and robust method in the ASR result verification. In the generalized word posterior probability (GWPP) [5][6] each spotted word is focused and all other word hypotheses in the graph or N-best list are treated as fillers. In the GWPP, words time boundaries constraints are relaxed, different word hypotheses may have different starting time and ending time. As the accurate information of the starting and the ending time of the phone can be obtained directly from the speech synthesis database, we can fix the time of the focused phone and use log likelihood ratio as a simplified version of GPP as our confidence score. Besides, using the starting and the ending time from the database avoids to do force alignment between phones thus greatly reduces the confidence score calculation time. Log likelihood ratio is defined as:

$$
\begin{gathered}
L L R=L L\left(C P_{0}\right)-\max _{i=1,2, \ldots, N}\left[L L\left(C P_{i}\right)\right] \\
L L\left(C P_{j}\right)=\log \left(p\left(O \mid \lambda_{j}\right)\right) /\left(t_{e}-t_{s}\right) \\
O=\left(o_{t_{s}}, o_{t_{s}+1}, \ldots, o_{t_{e}}\right) \\
j=0,1, \ldots, N
\end{gathered}
$$

Where $C P_{0}$ is the focused phone, $C P_{i}(\mathrm{i}=1, \ldots, \mathrm{N})$ is the $\mathrm{i}$ 'th hypotheses. $\operatorname{LL}\left(C P_{j}\right)(\mathrm{j}=0,1, \ldots, \mathrm{N})$ is the average $\log$ likelihood of the acoustic parameters $O$ from the starting frame $t_{s}$ to the ending frame $t_{e}$ to the according acoustic context dependent tri-phone HMM model. $p\left(O \mid \lambda_{j}\right)$ is the likelihood of the acoustic parameters observations $O$ to the focused phone's HMM model $(j=0)$ or the $j$ 'th hypotheses's tri-phone acoustic HMM model $(j>0)$. Since HMM model is used, force alignment within phone is needed to decide the boundaries of the states within an HMM model. Then 


$$
\begin{aligned}
& L L\left(C P_{j}\right)=\left[\sum_{k=1}^{M} \sum_{t=t_{s}}^{t_{e}} \log \left(p\left(O_{k} \mid \lambda_{k}\right)\right)\right] /\left(t_{e}-t_{s}\right), \\
& O_{k}=\left(o_{t_{s}}^{k}, o_{t_{s}+1}^{k}, \ldots, o_{t_{e}}^{k}\right) \\
& j=0,1, \ldots, N
\end{aligned}
$$

where $\mathrm{M}$ is the total state number of the HMM model, and $p\left(O_{k} \mid \lambda_{k}\right)$ is the likelihood of the acoustic parameters to the k'th state model of the focused phone's HMM $(j=0)$ model or the $\mathrm{j}$ 'th hypotheses's HMM model $(\mathrm{j}>0)$.

\section{4. heteronym annotation verification}

Since Mandarin is a tonal language, and heteronym can be pronounced differently either in the phone or just in tone, we divide the heteronyms into 2 categories. Class $\mathrm{A}$ is the heteronym pronounced differently just in tone, e.g. “当” (dang1, dang4). Class B is pronounced different in phone and either the same or different in tone, e.g. “凹”(ao1,wa1), “朝”(zhao1,chao2). We divide heteronym this way because the different pronunciation in the phone is a more prominent difference. From a heteronym dictionary in which commonly used heteronyms are recorded, a simple statistics is made: among all 154 commonly used heteronyms, 88 of them belong to Class A, and 76 belong to Class B.

For class A, since heteronyms are different just in tone, the problem becomes a tone recognition problem. As the tone bears close relationship with f0 features, we use log likelihood ratio of f0 to verify the annotation errors of class A. For class A, we generate 3 hypotheses by changing the current tone label of the focused phone from tone 1 to tone 4 (not including the original focused tone), and keeping the other context information unchanged.

For class B, both focused phone and its tone is changed to the phones with which the heteronym could possibly be mistaken to generate hypotheses, and the other context information also remains untouched.

For class A, F0 features are used to calculate the $\log$ likelihood ratio score. And for class B, both spectral features and the summation of spectral and $\mathrm{f0}$ features are used as a comparison. For each class, a global threshold is made, and heteronyms with the confidence score below the threshold are picked out as annotation errors.

\section{EXPERIMENTS}

\subsection{Corpus}

Since we don't have a testing corpus with enough heteronym errors labeled, we simulate erroneous test set by changing the Pinyin script of the recorded speech. For class $\mathrm{A}$, we modified randomly the tone of the heteronym from tone 1 to tone 4 on the Pinyin transcription. And for class B, both the phone and the tone of the heteronym is modified to its other possible pronunciations according to the heteronym dictionary in the Pinyin transcription to cause inconsistent between the script and the actual speech. For example, from the sentence "wei4mu3qin1men0pai2you1 jie3nan2"(为母 亲们排忧解难), In this sentence, “为” is a heteronym, and its tone is changed from tone 4 to tone 1 to make errors. "wei1mu3qin1men0 pai2you1jie3nan2" is randomly generated for class A errors. For class B, for example, we may change "tal tou2zhen3bu4qiang1lian3 chao2shang4tang3zhe 0" (他头枕步枪脸朝上躺着) to "ta1 tou2zhen3bu4qiang1lian3zhao1 shang4tang3zhe0", where “朝” is a heteronym.

We use a Mandarin speech synthesis database by a single standard speaker for evaluation. The corpus contain 7000 sentences. 1000 error free sentences (checked manually) containing 61395 phones are selected from the corpus for context information dependent tri-phone HMM training. Another 6000 sentences are selected for annotation modification and error evaluation. There are 9387 class A heteronyms and 4366 class B heteronyms in a total 6000 test sentences, and we "make" 1027 errors for class A and 483 errors for class B.

\subsection{Model used for testing}

1000 Mandarin sentences is used to train the context information dependent tri-phone HMM model. We computed 40 static LSFs and 1 dimension $\log$ fo feature with their first and second derivatives as the spectral and f0 acoustic feature. Long-term pitch normalization is used to normalize pitch in every $2 \mathrm{~s}$ duration before f0 model training and testing. HMM model with 5 no-skip states is trained for each context information dependent tri-phone as mentioned in section 3.1. Single mixture HMM is used because the context information dependent HMM model used is already fine enough, and also for the regard of the small amount of training data.

\subsection{Evaluation}

Receiver Operating Characteristic (ROC) is the usual way of presenting the utterance verification result. The ROC curve is the plot of a False Negative Rate (FNR) with respect to a False Positive Rate (FPR) at each threshold value. False positive means pronunciation errors haven't been detected and False negative means the correct pronunciations are judged as the wrong [6]. Normally, we choose the threshold that generate equal-error-rate (EER), i.e. $\mathrm{FNR}=\mathrm{FPR}$.

\subsection{Result}




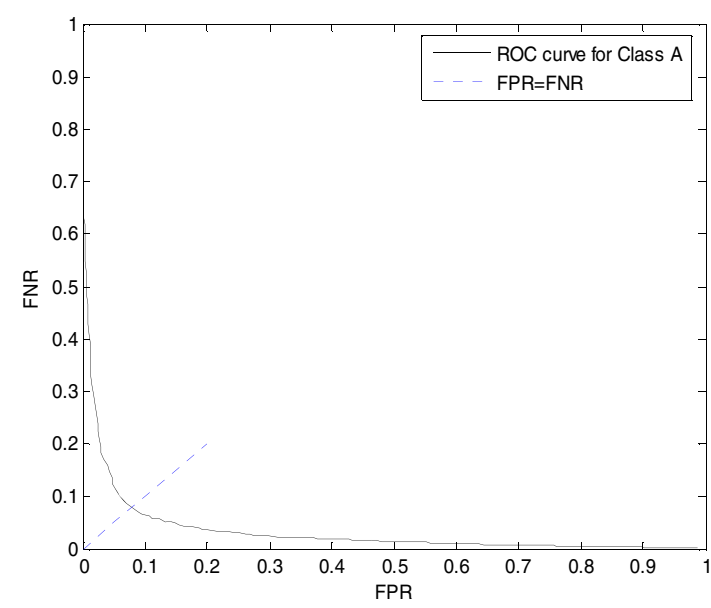

Figure 2: ROC curve for class A error

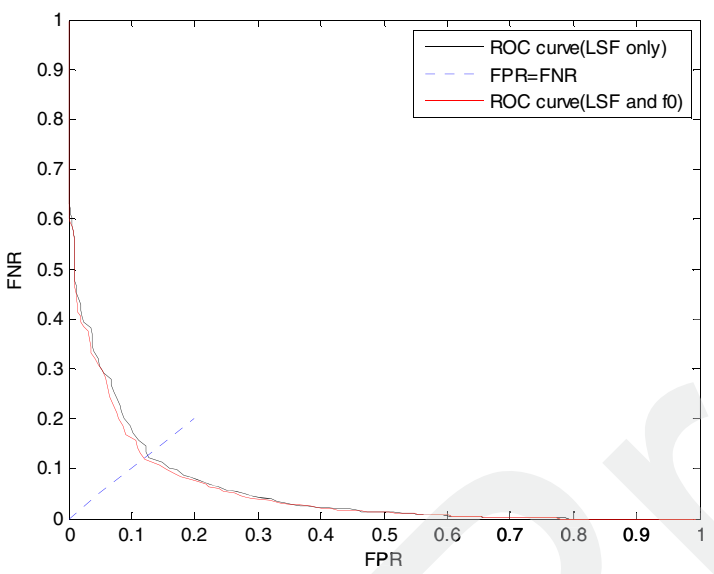

Figure 3: ROC curve for class B error

For class A, initial + final = syllable, because tones are carried by the finals, only polyphonic finals are used to calculate the log likelihood. The result is given in figure 2.The EER is $7.9 \%$ when the threshold is -0.7 . For class B, the average log likelihood of polyphonic syllable initial and final is used as confidence score. The ROC is shown in figure 3. The EER for Class B is $11.9 \%$. We tried both the $\log$ likelihood of LSF and the sum log likelihood of the LSF and $\mathrm{f} 0$ as confidence score for the class B error verification test and the result shows that using the sum gets a slightly better ROC curve. Both the LSF and the f0 are used in the force alignment within a phone to decide the state boundaries.

An other test for class A error detection on an actual database (the database had been checked manually many times thus contains little error) shows that, for a total of 13334 sentences containing 36098 heteronyms, 89 out of 123 class A errors are found out by checking 639 heteronyms. This greatly reduces the manual work. Because there is no errors list checked out manually for class B, tests for class $\mathrm{B}$ on the actual database errors is not conducted.

\section{CONCLUSIONS}

In this paper, we divide heteronyms into 2 categories and use the log likelihood of spectral and fo features as confidence measure to verify the annotation. For class A heteronyms, an EER of $7.9 \%$ is obtained, and for class B heteronyms, the EER is $11.9 \%$. As the same type tri-phone with context information HMM model is used both in our acoustic model training for speech synthesis and annotation verification, we can reduce the annotation verification system building time and labor cost. And the annotation verification system greatly reduces the manual works needed in checking the database by hand. Since heteronym annotation error is a big problem in MTTS automatic labeling, we can introduce this method as a fixed step in the speech synthesis system building to reduce the manual work in labeling..

\section{FUTURE WORK}

Since our goal is to improve the quality of the speech synthesis system and reduce the errors in the synthesized speech, error checking in the synthesis database is the first step of our work. Because our synthesis system is the HMM based speech concatenation system, we then will try to check the errors in the synthesized speech, so as to avoid selecting the unsuitable speech segments while concatenating the speech.

\section{REFERENCES}

[1] Chin-Hui Lee, Haizhou Li, Lin-Shan Lee, Ren-Hua Wang, Qiang Huo, "ADVANCES IN CHINESE SPOKEN LANGUAGE PROCESSING", World Scientific Publishing Co. Chapter 2E, 2006.

[2] Ingunn Amdal, Magne Hallstein Johnsen, Torbjorn Svendsen, "Log Likelihood Ratio Based Annotation Verification of a Norwegian Speech Synthesis Database,” NORSIG 2006.

Proceedings of the 7th Nordic. pp. 186-189, 2006.

[3] Yi Xu, "Production and perception of coarticulated tones", The Journal of the Acoustical Society of America, Volume 95, Issue 4, pp. 2240-2253, April 1994

[4] Koichi Shinoda, Takao Watanabe, "MDL-based contextdependent subword modeling for speech recognition", J.Acoust Soc. Jpn. (E) 21,2,(2000)

[5] Soong, F.K., Lo, W.K., and Nakamura, S. "Generalized word posterior probability (GWPP) for measuring reliability of recognized words," in Proc. SWIM-2004, Hawaii, January 2004. [6] L. Wang, Y. Zhao, M. Chu, F. K. Soong, and Z. Cao, “ Phonetic transcription verification with generalized posterior probability," in Proc. Eurospeech 2005, Lisboa, Portugal, pp. 1949 - 1952, 2005. 